\title{
Deep brain stimulation of the subthalamic nucleus transiently enhances loss-chasing behaviour in patients with Parkinson's Disease
}

\author{
Robert D. Rogers ${ }^{\text {a, }}$, Birgit Wielenberg b,c, ${ }^{\text {, Lars Wojtecki }}{ }^{\text {b,c }}$, Saskia Elben ${ }^{\text {b,c }}$, \\ Daniel Campbell-Meiklejohn ${ }^{\mathrm{d}}$, Alfons Schnitzler ${ }^{\mathrm{b}, \mathrm{c}, *}$ \\ a Department of Psychiatry, University of Oxford, South Parks Road, Oxford, OX1 3UD, United Kingdom \\ b Institute of Clinical Neuroscience and Medical Psychology, Heinrich-Heine-University Düsseldorf, Universitätsstrasse 1, 40225 Düsseldorf, Germany \\ ${ }^{c}$ Movement Disorders and Neuromodulation Unit, Department of Neurology, Heinrich-Heine-University Düsseldorf, Universitätsstrasse 1, 40225 Düsseldorf, Germany \\ d Department of Experimental Psychology, University of Oxford, South Parks Road, Oxford, OX1 3UD, United Kingdom
}

\section{A R T I C L E I N F O}

\section{Article history:}

Received 14 February 2011

Revised 19 May 2011

Accepted 18 June 2011

Available online 25 June 2011

\section{Keywords:}

Parkinson's Disease

Pathological gambling

Deep brain stimulation

Subthalamic nucleus

Reward

\begin{abstract}
A B S T R A C T
Dopaminergic treatments are associated with impulse control disorders such as pathological gambling in a subset of patients with Parkinson's Disease. While deep brain stimulation of the subthalamic nucleus has been reported to reduce symptoms of impulse control disorders in some Parkinson's Disease patients, little is known about its specific effects on gambling behaviour. In this experiment, we investigated the effects of deep brain stimulation of the subthalamic nucleus on one of the central features of pathological gambling: the tendency to chase losses. Loss-chasing is associated with impaired control over gambling behaviour and it is one of the most salient features of pathological gambling as it presents in the clinic. Twenty two patients with advanced idiopathic Parkinson's Disease and chronically implanted subthalamic nucleus electrodes for deep brain stimulation completed a simple laboratory model of loss-chasing behaviour twice: once with and once without stimulation. Exploratory analysis indicated that deep brain stimulation of the subthalamic nucleus increased the value of losses chased by patients with Parkinson's Disease when shifting from off- to on-stimulation. These effects were not attributable to changes in state affect or to the motor impairments produced by the withdrawal of deep brain stimulation of the subthalamic nucleus. The effects of the stimulation on the value of losses chased were more pronounced in female than in male patients and reduced in patients taking dopamine receptor agonists. Collectively, these results suggest that deep brain stimulation of the subthalamic nucleus can transiently alter the evaluation of accumulated losses during gambling episodes in idiopathic Parkinson's Disease.
\end{abstract}

(c) 2011 Elsevier Inc. All rights reserved.

\section{Introduction}

Accumulating evidence suggests that dopaminergic treatment is associated with patterns of behavioural addictions, such as impulse control disorders (ICDs) and the 'dopamine dysregulation syndrome' (Ferrara and Stacy, 2008), in a minority of patients with Parkinson's Disease (PD) (Dagher and Robbins, 2009; Lim et al., 2009; Weintraub et al., 2006). Such behaviour frequently involves excessive or pathological gambling (PG) (Driver-Dunckley et al., 2003), hypersexuality, spending, and the complex prolonged, but apparently purposeless, and stereotyped behaviour known as 'punding' (Evans

Abbreviations: PD, Parkinson's Disease; ICDs, impulse control disorders; STN, subthalamic nucleus; DBS, deep brain stimulation; PG, pathological gambling; LEU, levodopa equivalent units.

* Corresponding author at: Institute of Clinical Neuroscience and Medical Psychology, Heinrich-Heine-University Düsseldorf, Universitätsstrasse 1, 40225 Düsseldorf, Germany. Fax: + 492118118485.

E-mail address: SchnitzA@med.uni-duesseldorf.de (A. Schnitzler).

1 These authors made equal contributions to the experiment described here. et al., 2004). Risk factors for the development of PG in PD include male gender, relative youth at the time of diagnosis, a premorbid history of drug or alcohol abuse, depression, and elevated scores on the personality measure of novelty-seeking (Voon et al., 2007). Strikingly, the use of dopamine receptor agonists has been prominent in reported cases, while withdrawal of these treatments tends to produce an improvement in symptoms (Voon et al., 2006; Weintraub et al., 2006). However, relatively little is known about precisely which disturbances of mesostriatal circuitry, subsequent to nigral degeneration and dopamine replacement therapies, might promote PG in vulnerable PD patients (see Voon et al. (2010) for discussion).

Deep brain stimulation of the subthalamic nucleus (STN DBS) is widely used to treat PD and represents a clinically effective treatment for its motor symptoms (Krack et al., 2003). STN DBS has been reported to improve gambling problems in some PD patients (Ardouin et al., 2006; Bandini et al., 2007; Witjas et al., 2005) but, perhaps, exacerbates these problems in others (Lim et al., 2009; Smeding et al., 2007). Beneficial effects might be indirectly caused by modification of Parkinson's medication after surgery. But these clinical observations also raise the possibility that PG in PD is directly influenced by STN DBS 
and its effects upon its underlying reinforcement and response selection mechanisms (Ballanger et al., 2009; Frank, 2006; Frank et al., 2007a,b).

Continued gambling to recover losses - or loss-chasing - is a central behavioural feature of both social gambling and PG (American Psychiatric Association, 2000; Lesieur, 1977). Loss-chasing is strongly associated with impaired control over gambling behaviour and is central to, and the most significant step in the development, of PG (Lesieur, 1979). Descriptive theories of choice under uncertainty posit that gambling losses fall on the convex part of a psychophysical function relating nominal (e.g. monetary) values to their resultant subjective values or utilities, such that the decreases in utility associated with the possible bad outcomes of decisions to chase gambling losses are proportionately smaller than the substantial decreases in utility associated with the already accumulated losses (Kahneman and Tversky, 2000; Tversky and Kahneman, 1981). Under such circumstances, people will tend to choose to chase losses as opposed to simply accepting the certain losses already sustained.

While choosing to take extra risks to recover losses can be observed in everyday decision-making, and is a ubiquitous feature of light or social gambling (Dickerson et al., 1987; Kahneman and Tversky, 2000), it is markedly exaggerated in problem gamblers (Lesieur, 1979). Left unchecked, loss-chasing can produce a dangerous spiral of accelerating gambling involvement, increasing financial liabilities but diminishing resources to meet them, and the serious adverse family, social and occupational consequences (Corless and Dickerson, 1989).

Little is known about how STN DBS might influence clinically significant aspects of gambling behaviour in PD patients. Therefore, in this experiment, we investigated the effects of STN DBS on the performance of a laboratory model of loss-chasing behaviour that we have used in previous investigations to investigate the role of the fronto-striatal circuits in deciding to chase losses or quit during a run of losing gambles (Campbell-Meiklejohn et al., 2008) and to highlight the dissociable roles of dopamine and serotonin neuromodulation in these decisions (Campbell-Meiklejohn et al., 2010). Following other investigations of DBS in PD (Ballanger et al., 2009; Jahanshahi et al., 2000; Pillon et al., 2000), we used a within-subject, cross-over design to enhance our sensitivity for detecting treatment effects. Since we were interested in learning more about how STN DBS might influence loss-chasing as a behaviour that might promote excessive gambling, our PD patients were screened to exclude individuals with current significant gambling experience or PG. We tested the conjecture that STN DBS would modulate the manner in which such PD patients chase losses, either by influencing the number of decisions to keep gambling, or by influencing the values of losses that patients judged worth gambling to recover (Campbell-Meiklejohn et al., 2010).

\section{Materials and methods}

\section{Patients}

Ten male and twelve female patients with PD participated in this study which was approved by the ethics committee of University Hospital Duesseldorf and was carried out in accordance with the Declaration of Helsinki. All patients gave their written informed consent. All but one patient was right-handed. Demographic and clinical characteristics are set out in Table 1. The sample mean age of our patients was $65.55 \pm 1.72$ years (range: $43-78$ years), having been diagnosed for an average of $16.55 \pm 1.54$ years ( $7-34$ years). The mean number of years since STN surgery was $3.35 \pm 0.64$ years $(0.30-$ 10.0 years). Hoehn and Yahr (1967) ratings indicated predominantly Stage 2 disease while medicated and with STN DBS. Stimulation parameters for all 22 PD patents are listed in Supplementary Table 1.

Nineteen patients were being treated with levodopa medication. Sixteen of these patients were also receiving dopamine receptor agonists as follows: three were taking cabergoline, nine were taking pramipexole and four were taking ropinirol. One patient was using rotigotine. Twelve patients were receiving catechol-O-methyltransferase inhibitors: three tolcapone and nine entacapone. The mean daily levodopa equivalent units (LEU) were $639.08 \pm 79.96 \mathrm{mg}(0-1349)$. Finally, five patients were receiving the NMDA antagonist amantadine. Four of the six patients not being treated with dopamine receptor agonists had exhibited symptoms of ICDs when treated with these medications previously; however, these symptoms had not included excessive or problematic patterns of gambling behaviour.

All patients were assessed using a semi-structured SCID-I interview (First et al., 2002) to exclude current DSM-IV major depression (American Psychiatric Association, 2000). Five patients in the sample were taking anti-depressants (amitriptyline, venlafaxine, mirtazapine, citalopram, or escitalopram). However, our sample's mean score on the Beck Depression Inventory (Beck et al., 1961) was only $4.36 \pm 0.73$ (012). One patient's score of 12 was above the threshold of 10 which has been used to identify mild-to-moderate depressive symptoms in German populations (Hautzinger et al., 1995). However, exclusion of this patient from our data analysis made no difference to the statistical significance of the results reported below.

Patients also completed a self-report measure of manic symptoms over the previous 14 days (Shugar et al., 1992). One patient's score of 14 was equal to the cut-off score for 'caseness' in German populations (Schöttle et al., 2006). Again, however, exclusion of this patient did not change the statistical significance of our findings. Overall, the mean mania rating of our sample was relatively low at $6.68 \pm 0.94$ (0-14).

Different patients were taking a variety of central nervous system active drugs for other indications: one patient was receiving the $\mathrm{D}_{2}$ dopamine receptor antagonist, sulpiride, to treat vertigo; two others were receiving the atypical antipsychotic, quetiapine; one patient was taking the cholinesterase inhibitor, rivastigmine; two patients, the benzodiazepine derivatives, clonazepam and tetrazepam; one patient was being treated with the non-benzodiazepine hypnotic, zopiclone; and one patient, with the mood-stabiliser, carbamazepine.

Previous clinical investigations have suggested a score of 130 or below on the Mattis Dementia Rating (Schmidt et al., 1994) as an appropriate threshold for dementia in PD patients (Witt et al., 2008). None of the patients in our experiment scored below this threshold; the sample mean being $140.27 \pm 0.64$ (134-144).

Punding behaviours were assessed informally using the questions listed in the instrument described by Evans et al. (2004). None of the patients described consistent patterns of the complex prolonged, purposeless, and stereotyped behaviour that have been noted in previous investigations of PD patients (Lawrence et al., 2007). Finally, all patients completed the South Oaks Gambling Screen (Lesieur and Blume, 1987). All scores were 0 , reflecting the absence of gambling problems. In general, gambling participation was confined to occasional lottery play, or informal card games with family or friends for small stakes.

\section{Design}

The study consisted in a within-subject, cross-over design. Patients completed the loss-chasing game once with STN DBS ('STN DBS-on') and once without STN DBS ('STN DBS-off'). Ten patients completed the STN DBS-on condition first and the STN DBS-off condition second; the remainder completed these conditions in the reverse order.

\section{Loss-chasing game}

Our loss-chasing game suitable has been described by CampbellMeiklejohn et al. (2008) (Campbell-Meiklejohn et al., 2010). On each play of the game, patients were required to choose between gambling to recover a loss (at the risk of doubling its size) or quitting (and surrendering a certain loss). Such dilemmas consistently induce risky 
Table 1

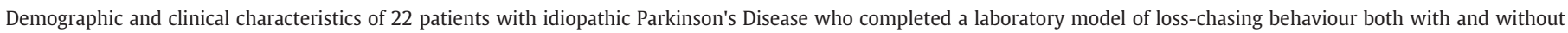
subthalamic deep brain stimulation (STN-DBS).

\begin{tabular}{|c|c|c|c|c|c|c|c|c|c|c|c|}
\hline Participant & Gender & Age & Dominant-type & $\begin{array}{l}\text { Duration of } \\
\text { illness (years) }\end{array}$ & $\begin{array}{l}\text { Hoehn } \\
\text { and Yahr }\end{array}$ & $\begin{array}{l}\text { L-Dopa } \\
\text { equivalent }\end{array}$ & $\begin{array}{l}\text { Time since } \\
\text { surgery (years) }\end{array}$ & $\begin{array}{l}\text { Mattis } \\
\text { score }\end{array}$ & $\begin{array}{l}\text { BDI } \\
\text { score }\end{array}$ & $\begin{array}{l}\text { Mean value of } \\
\text { losses chased } \\
\text { ON }\end{array}$ & $\begin{array}{l}\text { Mean value of } \\
\text { losses chased } \\
\text { OFF }\end{array}$ \\
\hline 1 & Female & 71 & Akinetic/rigid & 20 & 3.00 & 345 & 10.00 & 134 & 3 & 91,3 & 69,39 \\
\hline 2 & Female & 71 & Aquivalence & 8 & 2.00 & 861 & 1.00 & 142 & 6 & 150,42 & 169,49 \\
\hline 3 & Male & 70 & Tremor/dominant & 12 & 2.00 & 517 & 5.00 & 140 & 2 & 185,22 & 133,45 \\
\hline 4 & Male & 48 & Tremor/dominant & 15 & 2.00 & 813 & 5.00 & 142 & 4 & 128,24 & 107,89 \\
\hline 5 & Male & 43 & Tremor/dominant & 10 & 2.00 & 100 & 3.00 & 140 & 7 & 181,21 & 165,68 \\
\hline 6 & Male & 68 & Akinetic-rigid & 19 & 2.50 & 1154 & 8.00 & 134 & 3 & 27,27 & 100,83 \\
\hline 7 & Male & 66 & Akinetic-rigid & 24 & 2.50 & 1349 & 6.00 & 136 & 2 & 140,73 & 171,71 \\
\hline 8 & Male & 72 & Akinetic-rigid & 14 & 2.00 & 1140 & 1.00 & 143 & 7 & 92,69 & 86,67 \\
\hline 9 & Female & 63 & Akinetic-rigid & 12 & 1.00 & 633 & 1.00 & 144 & 12 & 162,68 & 80 \\
\hline 10 & Male & 58 & Akinetic-rigid & 16 & 2.00 & 308 & 1.00 & 144 & 1 & 157,95 & 150,31 \\
\hline 11 & Female & 66 & Akinetic-rigid & 14 & 2.00 & 368 & 0.30 & 140 & 6 & 95,17 & 76,5 \\
\hline 12 & Female & 74 & Akinetic-rigid & 15 & 2.00 & 50 & 0.30 & 137 & 0 & 148,89 & 97,65 \\
\hline 13 & Female & 64 & Akinetic-rigid & 16 & 2.00 & 736 & 2.00 & 143 & 0 & 0 & 0 \\
\hline 14 & Female & 78 & Akinetic-rigid & 34 & 2.00 & 665 & 9.00 & 142 & 3 & 102,31 & 83,68 \\
\hline 15 & Female & 74 & Akinetic-rigid & 18 & 2.00 & 1318 & 4.00 & 143 & 0 & 169,49 & 87,5 \\
\hline 16 & Female & 64 & Akinetic-rigid & 25 & 2.00 & 651 & 7.00 & 141 & 0 & 88 & 78,7 \\
\hline 17 & Male & 63 & Tremor/dominant & 7 & 2.00 & 0 & 2.00 & 139 & 9 & 127,14 & 107,69 \\
\hline 18 & Female & 71 & Tremor/dominant & 30 & 2.00 & 534 & 3.00 & 141 & 6 & 130 & 119,47 \\
\hline 19 & Male & 62 & Akinetic-rigid & 8 & 2.50 & 508 & 1.00 & 137 & 5 & 208 & 180,67 \\
\hline 20 & Female & 65 & Akinetic-rigid & 14 & 2.00 & 597 & 2.00 & 143 & 3 & 100,54 & 111,79 \\
\hline 21 & Female & 68 & Akinetic-rigid & 16 & 2.00 & 766 & 1.00 & 141 & 10 & 29,41 & 52 \\
\hline 22 & Male & 63 & Akinetic-rigid & 17 & 2.00 & 650 & 1.00 & 140 & 7 & 169.49 & 150.81 \\
\hline
\end{tabular}

choices in a variety of social and economical contexts (Shafir and Tversky, 1995). Descriptive theories of choice attribute this behaviour to the proportionately smaller reductions in subjective value or utility associated with unsuccessful decisions to chase compared to the reductions in utility associated with already-sustained losses (Kahneman and Tversky, 2000). We have observed that gambling to recover losses in our game is positively associated with psychometric measures of the tendency to chase loss in other gambling situations (CampbellMeiklejohn et al., 2008).

At the start of the game, the patients were told that they had a notional $€ 20,000$ to play with, but that the patient with the most points at the end of the experiment would win a real prize of $€ 100$. On each 'round' of the game, $€ 10, € 20, € 40$, $€ 80$ or $€ 160$ was subtracted from the game total. This amount appeared below the choices: 'Quit' and 'Play' (Fig. 1). At this point, the patients could choose to 'Quit', accepting this loss and ending the round immediately ('quit-loss' outcome), or they could choose to 'Play', that is, chase the loss. Thus, they could gamble on recovering an amount equal to the loss but at the risk of increasing their losses by the same amount. If the outcome of a decision to gamble was positive ('chase-win' outcome), the loss was recovered and the round ended. If the outcome was negative ('chase-loss' outcome), the loss was doubled and patients were given another chance to quit or to chase the doubled loss in the next choice of the round. The options for each choice 'Play' or 'Stop' - appeared equally often towards the left-hand and righthand sides of the choice displays.

Outcome displays indicated whether patients had won a gamble and that no money was lost ('chase-win'); whether they had lost a gamble and the size of the doubled loss ('chase-loss'); or the amount lost if patients chose to quit the round ('quit-loss'). At the end of each round, the patients were also informed about their final losses in a 'round-loss' display. This display indicated the total cumulative losses for that round, in red text if the losses were greater than 0 but in green text if the losses equalled 0 . Six rounds began with losses of $€ 10, € 20$, $€ 40$, $€ 80$ or $€ 160$, yielding 30 rounds. If patients continued losing over a round, losses kept doubling until they reached $€ 640$, at which point the round ended having incurred this maximum loss.

The outcomes of the loss-chasing game were distributed such that 18 rounds would eventually return all losses if the patients decided to play on every choice of the game. However, 12 rounds would result in the maximum loss of $€ 640$. Chase-win outcomes were positioned randomly within each round such that a winning outcome would occur equally often after any number of (between 1 and 6 ) consecutive losses. In order to discourage patients from adopting conservative strategies involving early and persistent decisions to quit (so as to preserve as much of the play money as possible), no information was provided about their diminishing total of play money during the game. Patients were also informed that they would not achieve their best possible score by exclusively playing or by exclusively quitting.

In summary, the patients in our experiment were confronted with dilemmas involving a choice between gambling to recover a loss at the risk of doubling its size, or surrendering the loss and ending the chase, while at the same time preserving as effectively as possible the resources that allowed play to continue. The value of this nominal reward was provided by the context of an inter-patient competition for a real monetary prize. This method of mixed nominal and actual reward has been used in economics to demonstrate behaviour qualitatively and quantitatively similar to that observed outside the laboratory (Cubitt et al., 1998).

\section{Dependent measures}

Only those rounds of the loss-chasing game that terminated with a decision to quit or provision of the maximal loss ( $€ 640)$ were included in statistical analyses; i.e. all rounds of the game that ended with a win were excluded. In this way, the analysed loss-chasing episodes reflected patients' intentions to keep playing during a run of losing outcomes (i.e. reflecting how far the patients would go without the random variation of winning outcomes). This data provides representative measures of the number and magnitudes of losses that were acceptable to patients before they decided to quit during a chasing episode.

Our loss-chasing game provided the following dependent measures: a) proportion of choices to gamble out of all choices made during the game; b) mean number of losses chased per round (consecutive decisions); c) mean value of accumulated losses associated with decisions to chase; d) mean value of accumulated losses associated with decisions to quit. We also measured the mean deliberation times required when deciding to chase and to quit. 


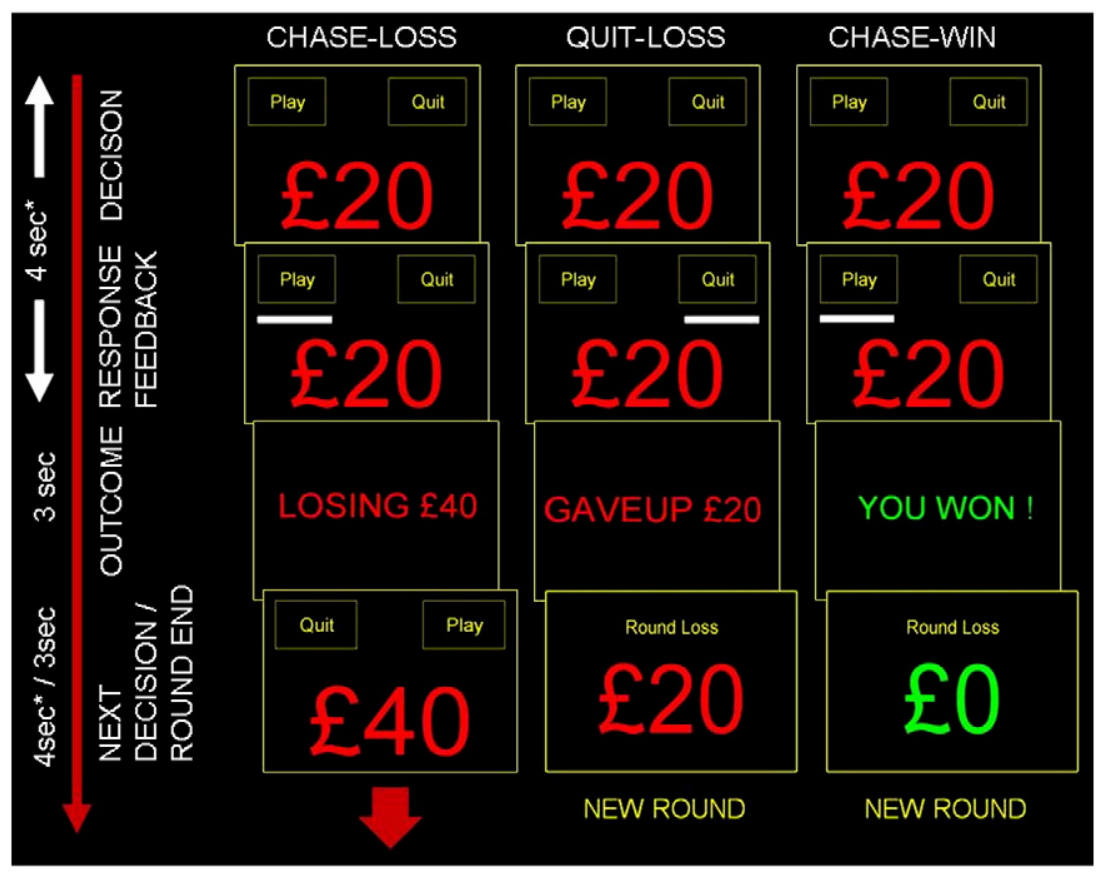

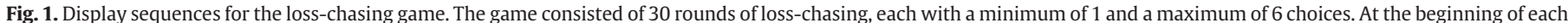

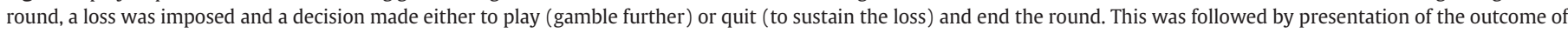

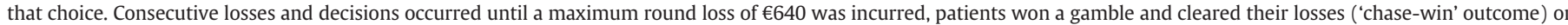

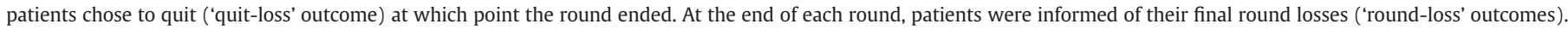

\section{Procedure}

Each patient completed the protocol in a single study session of $2.5 \mathrm{~h}$, as part of an annual DBS stimulation assessment. All patients were tested while taking their usual disease medications in a defined medication-on state. Self-reported state positive and state negative affect (as scored by the PANAS) (Watson et al., 1988) was measured just prior to completing the loss-chasing game in the STN DBS-on condition and in the STN DBS-off condition. An interval of 30 min was allowed to elapse between turning patients' stimulation off and completion of the loss-chasing task in the STN DBS-off condition.

Motor symptoms associated with the 2 stimulation conditions were investigated in separate clinical sessions using the Unified Parkinson's Disease Rating Scale (UPDRS) III motor scale (Fahn and Elton, 1987). Patients' motor scores were examined under conditions involving the following 3 combinations of dopaminergic medication and STN DBS: medication-on/STN DBS-on; medication-off/STN DBSoff and; medication-off/STN DBS-on.

\section{Statistical analyses}

The effects of STN DBS upon state affect and the dependent measures of our model of loss-chasing were tested using repeated measures analysis of variance (ANOVA) with the between-subject factors of gender and order of condition (STN DBS-on/STN DBS-off vs STN DBS-off/STN DBS-on) and the within-subject factor of condition (STN DBS-on vs STN DBS-off). Our analyses demonstrated significant interactions between DBS condition and order of condition. These interactions were examined by testing the significance of simple effects of STN DBS condition for the first and second completion of the loss-chasing game.

Motor symptoms, as measured by the UPDRS III motor scores, were tested by repeated measures ANOVA with the between-subject factor of gender and a single within-subject factor having the 3 combinations of dopaminergic medication and DBS (medication-on/STN DBSon, medication-off/STN DBS-off vs medication-off/STN DBS-on). The significance of pair-wise differences between these combinations was tested with post-hoc $t$-tests.

Standard linear regression was used to explore the relationship between the proportionate changes in our dependent measures in the STN DBS-on compared to the STN DBS-off condition on the one hand and demographic, clinical and experimental regressors on the other hand. Categorical (and binary) regressors included order of condition (with 'STN DBS-off/STN DBS-on' as the reference), gender (with 'male' as the reference), and the use of dopamine receptor agonists, amantadine and anti-depressant treatments (with 'no use' as the reference in these cases). Continuous regressors (standardised as Z-scores) included years since diagnosis, BDI score, and mania rating (scored over 14 days) and proportionate change in the UPDRS III motor scores, acquired during a separate clinical assessment, in the medication-off/STN DBS-on compared to the 'medication-off/STN DBS-off' combination.

\section{Results}

\section{State affect and motor function}

State positive affect was not significantly elevated or diminished in the STN DBS-on compared to the STN DBS-off condition $(22.41 \pm 1.67$ vs $23.64 \pm 1.64), F(1,18)=1.19$. State negative affect was not significantly increased $(12.73 \pm 0.74$ vs $13.14 \pm 0.66), F<1$. There were no reliable interactions involving STN DBS, gender or order of condition, all $\mathrm{F}(1,17)<1.79$.

UPDRS III motor scale scores differed depending upon whether patients were assessed on and off medication, and with and without STN DBS (see Supplementary Table 2), F(2, 19) =76.93, p<0.0001. Post-hoc tests demonstrated significantly lowered motor symptoms during the medication-on/STN DBS-on compared to the medicationoff/STN DBS-off condition (15.86 \pm 1.31 vs $41.14 \pm 2.35), \mathrm{t}(21)=$ $-12.00, \mathrm{p}<0.0001$, and the medication-off/STN DBS-on condition $(24.50 \pm 1.61), \mathrm{t}(21)=-8.34, \mathrm{p}<0.0001$. Motor symptoms were reliably lower in the medication-off/STN DBS-on compared to medication-off/STN DBS-off condition, $\mathrm{t}(21)=8.73, \mathrm{p}<0.0001$. 


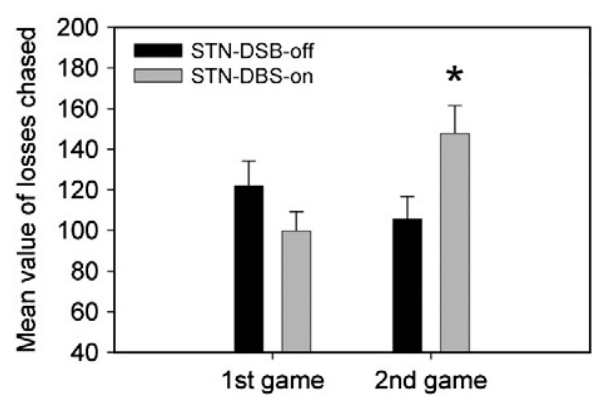

Fig. 2. Loss-chasing with and without deep brain stimulation of the subthalamic nucleus (STN DBS) in 22 patients with idiopathic Parkinson's Disease. The values of losses chased are shown separately for the 1st and 2nd completion of the game. Interaction between condition (STN DBS-on vs STN DBS-off) and order (STN DBS-off/ STN DBS-on vs STN DBS on/STN-DBS-off): F(1, 17) $=6.65, \mathrm{p}<0.05$; *simple effect, 2nd game: $\mathrm{F}(1,18)=6.30, \mathrm{p}<0.05$.

\section{Loss-chasing game}

\section{Proportionate choice}

In general, STN DBS produced different effects on loss-chasing behaviour during the first occasion that patients completed the game compared to the second occasion, reflected in significant or nearsignificant 2-way interactions between STN DBS condition and the order of condition. While STN DBS reduced the proportion of decisions to chase made during the first completion of the loss-chasing game $(0.49 \pm 0.08$ vs $0.60 \pm 0.09$ ), it tended to increase them during the second completion $(0.71 \pm 0.08$ vs $0.52 \pm 0.08), F(1,18)=3.94, p=0.063$. Similarly, while the number of the consecutive decisions to chase was reduced in the STN DBS-on compared to STN DBS-off conditions during the first completion of game ( $1.05 \pm 0.26$ vs $1.44 \pm 0.29$ ), they tended to be increased in the STN DBS-on condition on the second occasion $(1.86 \pm 0.34$ vs $1.15 \pm$ $0.28), \mathrm{F}(1,18)=3.22, \mathrm{p}=0.09$.

\section{Value of losses chased/surrendered}

STN DBS had reliably different effects on the value of losses chased on the 2 completions of the game, demonstrated by a significant 2way interaction between DBS condition and the order, $F(1,17)=6.65$, $\mathrm{p}<0.05$. Analysis of the simple effects showed that STN DBS reduced slightly the magnitude of losses chased during the first occasion that patients played the game, $F(1,17)=1.41$, but then significantly increased the size of losses chased on the second occasion (see Fig. 2), $\mathrm{F}(1,18)=6.30, \mathrm{p}<0.05$. Similarly, STN DBS reduced the value of losses surrendered when deciding to quit chasing losses on the second occasion that the loss-chasing game was played $(72.39 \pm 14.18$ vs $107.21 \pm 9.67)$ compared to the first occasion $(106.53 \pm 10.75$ vs $101.07 \pm 15.17$ ); however, the two way interaction between condition and order was not statistically significant, $\mathrm{F}<1$.

\section{Mean deliberation times}

The time needed by patients to decide to chase losses was slightly reduced in the STN DBS-on condition compared to the STN DBS-off condition ( $2859 \pm 370$ vs $4263 \pm 902 \mathrm{~ms}$ ); however, this difference was not significant, $\mathrm{F}(1,17)=1.45$; moreover, deliberation times for chasing losses were not markedly altered by STN DBS on the first occasion that the game was completed ( $2705 \pm 318$ vs $4252 \pm 1156 \mathrm{~ms}$ ) compared to the second time $(2974 \pm 615$ vs $4277 \pm 1163 \mathrm{~ms}), \mathrm{F}<1$. Finally, deliberation times associated with decisions to quit were broadly comparable in the 2 DBS conditions ( $3992 \pm 892$ vs $3423 \pm 504 \mathrm{~ms}$ ), $\mathrm{F}<1$; there were no marked DBS-related changes on the first ( $4481 \pm$ 1589 vs $3744 \pm 857 \mathrm{~ms}$ ) or second time ( $3067 \pm 733$ vs $3744 \pm 504 \mathrm{~ms}$ ) that the game was completed, $\mathrm{F}<1$.
Table 2

Multivariate regression of proportionate increase in the value of losses chased during a loss-chasing game in the STN-DBS-on condition compared to the STN-DBS-off condition against gender (with 'male' as the reference), order of condition (with 'STN-DBS-on/ STN-DBS-off as the reference), years since diagnosis, proportionate change in the UPDRS-III motor score in the 'medication-off/STN-DBS-on' compared to the 'medication-off/STN-DBS-on' combination (separate clinical assessment), BDI score, mania rating (over previous 14 days), and use of antidepressants and dopamine receptor agonists and use of amantadine ('no use' as reference).

\begin{tabular}{lrrrrrrr}
\hline & \multicolumn{2}{l}{ Unstandardised } & & \multicolumn{2}{c}{ Standardised } & & \\
\cline { 2 - 3 } Regressor & $\beta$ & S.E. & & $\beta$ & & t-test & p-value \\
\hline Intercept & 0.589 & 0.173 & & & 3.405 & 0.005 \\
Order & 0.503 & 0.149 & & 0.657 & & 3.385 & 0.005 \\
Gender & 0.586 & 0.152 & & 0.771 & & 3.861 & 0.002 \\
Years of dis. & 0.046 & 0.075 & & 0.121 & & 0.613 & 0.551 \\
BUPDRS-III & 0.057 & 0.079 & & 0.148 & & 0.717 & 0.487 \\
BDI score & -0.067 & 0.091 & & -0.170 & & -0.742 & 0.473 \\
Mania & -0.113 & 0.065 & & -0.288 & & -1.731 & 0.109 \\
Antidepressants & -0.389 & 0.190 & & -0.463 & & -2.040 & 0.064 \\
Dopamine agonists & -0.569 & 0.199 & & -0.678 & & -2.866 & 0.014 \\
Amantadine & 0.002 & 0.179 & & 0.002 & & 0.012 & 0.990 \\
\hline
\end{tabular}

Regressions

We regressed the proportionate changes in the value of losses chased during the performance of our loss-chasing game in the STN DBS-on condition relative to STN DBS-off condition against order of condition (with 'STN DBS-on/STN DBS-off' as the reference), years since diagnosis, the proportionate change in the UPDRS III motor score (in the 'medication-off/STN DBS-on' compared to medication-off/STN DBS-off combinations), BDI score, mania rating (over the last 14 days), and use of dopamine receptor agonists, amantadine and anti-depressants (with 'no use' as reference for these regressors). Gender was not equally distributed across those participants who completed the STN DBS-on condition first those who completed it second ( 4 females in the former compared to 8 females in the latter). Therefore, we also included gender as a regressor (with 'male' as a reference).

The intercept of the model was significant, indicating that the value of losses chased was significantly increased during STN DBS-on compared to the STN DBS-off condition (see Table 2) $(\mathrm{p}<0.01)$. However, the coefficient terms demonstrate that this effect was moderated by other factors. As expected, completing the game without STN DBS on the first occasion but with STN DBS on the second occasion was associated with larger effects of STN DBS on losses chased $(p<0.01)$. STN DBS also increased the size of losses chased to a significantly greater extent in female compared to male patients (Fig. 3) $(\mathrm{p}<0.01)$. By contrast, the use of dopamine agonists was associated with smaller effects of STN DBS on the values of losses
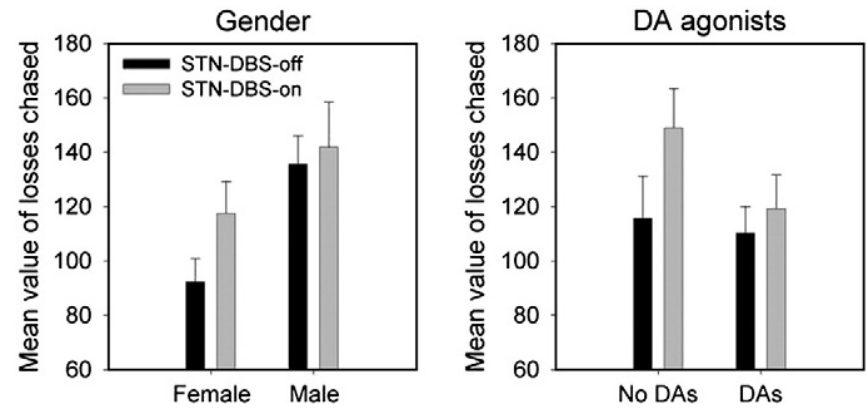

Fig. 3. Loss-chasing with and without deep brain stimulation of the subthalamic nucleus (STN DBS) in 22 patients with idiopathic Parkinson's Disease. The values of losses chased are shown separately for male and female patients, and with and without the dopamine (DA) agonists. Interaction of gender and condition (STN DBS-on vs STN DBS-off): $\beta=0.625(0.144), p=0.001$; interaction of DA agonists and condition: $\beta=$ -0.550 (0.169), $\mathrm{p}<0.01$. 
chased $(p<0.05)$ while a similar trend was apparent for the use of anti-depressants $(p=0.06)$. Patients' changes in the UPDRS across stimulation conditions, recent depressive and manic symptoms, use of amantadine and years since diagnosis, were not significant predictors of the effects of STN DBS upon values chased during performance of our loss-chasing game.

\section{Discussion}

The tendency to keep gambling in order to recover previous losses loss-chasing behaviour - is common in everyday decision-making as well in recreational gamblers (Dickerson et al., 1987). However, this behaviour is markedly exaggerated in problem gamblers and constitutes a significant mechanism in generating the adverse of life consequences of PG (Dickerson et al., 1987; Lesieur, 1977). Our results indicated that STN DBS in patients with idiopathic PD did not reduce the tendency to chase losses, but rather increased the value of losses that patients are willing to play for when shifting from the STN DBS-off to DBS-on conditions. STN DBS also produced larger increases in the values of losses chased in female compared to male patients, but smaller changes in patients who were using dopamine receptor agonists. Before considering the significance of these findings, we consider some methodical issues.

First, these findings are not attributable to short-term changes in emotional state that have been associated with STN DBS (Okun et al., 2003; Stefurak, et al., 2003). During the study visit, we observed only marginal changes in state positive affect or negative affect in the STN DBS-on compared to the STN DBS-off condition. Moreover, the time needed for patients to make their decisions to stop chasing losses was similar in both conditions. A previous study suggested that STN DBS reduces the ability to delay responding when faced with decision conflict (Frank, 2006). Consistent with this possibility, we observed that decisions to chase losses during the STN DBS-on condition tended to be faster compared to the STN DBS-off condition. However, this difference was not statistically significant.

Second, we acknowledge that the experimental paradigm used to model loss-chasing behaviour in our experiment has an abstracted quality and does not fully capture the excitement associated with real gambling activities, such as those that might come to be problematic in a minority of PD patients (Weintraub et al., 2006). However, consistent with previous experiments (Campbell-Meiklejohn et al., 2008; Tversky and Kahneman, 1981), the decisions made by our patients over the course of the game were in line with the predictions of descriptive models of the relationship between losses and subjective value (Kahneman and Tversky, 1979; Tversky and Kahneman, 1981). Moreover, we have previously observed that the proportion of decisions to chase during our loss-chasing game is positively associated with the tendency to chase in real-life gambling situations (Campbell-Meiklejohn et al., 2008). These observations provide some reassurance that our game captures some of the cognitive and emotional processes underlying loss-chasing behaviour. Our findings also demonstrate advantages of laboratory measures of clinically salient behaviours, such as gambling, over self-report (pre- and post surgical) questionnaires.

Third, a final concern may involve the lack of an age-matched control sample without PD which might have been useful in supplying some normative data about the degree to which individuals in this age range (mid-60s), and with this minimal amount of gambling experience, would chase losses in the context of our loss-chasing game. However, our purpose was not to detect changes in gambling behaviour in patients with STN DBS compared to the wider population of PD patients. Rather, we sought to test whether STN DBS would alter the loss-chasing of patients selected to have no, or negligible, gambling experience, as a way of learning more about the role of STN DBS in the gambling behaviour of PD patients. Our design is similar to others in which the critical comparisons have involved cognitive function when the STN DBS is applied and when it is withheld (Ballanger et al., 2009; Jahanshahi et al., 2000; Pillon et al., 2000). These designs use each patient as their own control and are highly sensitive to the kind of subtle behavioural changes we report here. In fact, the overall proportion of decisions to chase in our sample in the STN DBS-off condition, $0.56 \pm 0.06$, is comparable to the average proportion of 3 younger samples (late-20s) we have recently collected using the same game, $0.62 \pm 0.04$ (Campbell-Meiklejohn et al., 2010). Therefore, there is little reason to suppose that the chasing behaviour of our sample is unrepresentative of the larger population of patients with PD.

Finally, we note that the effects of STN DBS on loss-chasing were subtle, being most apparent in the values of losses judged worth chasing, rather than the proportion of decisions to chase, and in those circumstances in which patients had already completed the loss-chasing game once; they were also larger in female compared to male patients. These results reflect an exploratory, rather than hypothesis-testing analytic approach. Additionally, our findings that order of DBS condition, gender and dopamine agonist treatment had divergent influences upon the effects of STN DBS on loss-chasing must be treated as preliminary because of inter-correlations between regressors (e.g. gender) and because our multivariate regression would have limited statistical power in a small sample of patients. However, notwithstanding these precautions, our data highlight new avenues for research into the relationship between STN DBS in PD and the gambling problems observed in a minority of patients (Voon et al., 2007).

The most dramatic finding of our experiment is that STN DBS increased the value of losses chased during the second occasion that the loss-chasing game was completed, and not during the first occasion. This is surprising in the light of influential proposals that the STN inhibits or suspends selected actions before being enacted while a 'brake' is applied to allow alternative candidate actions to be more appropriately considered or appraised (Frank, 2006; Frank and Claus, 2006; Swann et al., 2011). Still other neuropsychological investigations have suggested that STN DBS impairs aspects of conditional associative learning and verbal learning (Heo et al., 2008; Saint-Cyr et al., 2000). All of this evidence might suggest that STN DBS should have had its largest impact on the loss-chasing while patients were unfamiliar with the game. However, we found the precise opposite result: STN DBS altered loss-chasing only when patients had completed the game once previously.

There are two possible explanations: First, our data are consistent with other findings that STN lesions following the acquisition of Pavlovian approach response towards food induces subsequent sensitivity to the omission of reward (Winstanley et al., 2005). Speculatively, our data suggest that once the loss-chasing game had been learnt in the first completion of the game, STN DBS altered the value of accumulated losses in a way that facilitated continued gambling. As noted above, decisions to chase losses arise because the relationship between monetary losses and their subjective values is described by a convex psychophysical function (Kahneman and Tversky, 1979; Tversky and Kahneman, 1992). According to this function, the decreases in utility associated with the bad outcomes of continued play are proportionately smaller than the decreases in utility arising out of the certain losses sustained already; in other words, as the losses mount, the penalty in further diminutions in utility are continuously reduced. Our finding that STN DBS increases the size of losses chased in our gambling game suggest that subjective evaluation of the further losses that might result is reduced.

Second, the observation that increased values of losses chased during STN DBS-on compared to STN DBS-off were pronounced during the second occasion also suggests different effects of STN DBS depending on duration of stimulation. Patients who completed the loss chasing game in the STN DBS-on condition first had received uninterrupted stimulation up until the stimulation was removed for 30 min prior to the second completion of the game. By contrast, patients who played the loss chasing game in STN DBS-on condition 
second received stimulation for only 30 min, raising the possibility that the reinstatement of stimulation for a short interval prompted the increase in values chased by these patients. This possibility is strengthened by observations that impulsive behaviours, including patient suicides, are most frequent following the commencement of DBS treatment, perhaps reflecting short-term disturbances to that can be stabilised overtime (Voon et al., 2008). Investigations of these possibilities could be done with designs in which stimulation is applied (or removed) and then its effects upon loss-chasing measured over several successive completions of the game.

\section{Clinical factors in the effects of STN DBS on loss-chasing behaviour}

Estimating the clinical effects of STN DBS on PG and other compulsive or impulsive behaviours is also complicated by postsurgical reductions in dopamine receptor agonist treatments. Our study identifies demographic and clinical factors that may influence, or mask, the effects of STN DBS on gambling behaviour. First, the effects of STN DBS on the values of losses chased were greater in female patients with PD than in male patients. Clinical studies have demonstrated significant gender differences in gambling behaviour (Cregh-Tyte and Lepper, 2004), with other evidence suggesting greater preferences for risk-averse choices in female compared to males (Rosen et al., 2003). It is striking, therefore, that the higher value of losses chased in the STN DBS-on condition seen in the female patients was associated with markedly lower values chased during the STN DBS-off condition (Fig. 3). By contrast, the reduced effects of STN DBS in the male patients were observed in the context of relatively higher losses gambled for in both the STN DBS-off and STN DBS-on conditions. This suggests that the larger effects of STN DBS in the female patients reflect the lower baseline against which the STN DBS effects were expressed.

Second, we found that patients who were not using dopamine receptor agonists (and, at trend level, patients who were not using antidepressants) chased smaller losses in the STN DBS-off condition but clearly larger losses in the STN DBS-on condition, while patients who were receiving dopamine receptor agonist treatments tended to chase for comparable losses in both conditions (Fig. 3). Treatments with dopamine receptor agonists have been shown to retard PD patients' ability to learn from losing outcomes during the performance of probabilistic decision-making tasks, but enhance learning from winning outcomes (Frank et al., 2007a,b; van Eimeren et al., 2009; Voon et al., 2010). There is also recent evidence that the effects of treatments with dopamine receptor agonists on learning from losing outcomes are reduced in PD patients with a history of PG or compulsive shopping behaviours (Voon et al., 2010). At first glance, our data suggest that treatments with dopamine receptor agonists block the increase in the values chased associated with STN DBS in PD patients with limited gambling histories. However, the patients without dopamine receptor agonists were previously withdrawn from treatment with dopamine receptor agonists precisely because of the emergence of symptoms associated with impulse control disorders. Thus, it may be that the increased loss chasing behaviour observed during STN DBS-on in patients without dopamine receptor agonists is related to pre-existing vulnerabilities to develop ICDs following these treatments in a minority of patients recruited to our study.

Further experiments will be needed to replicate and understand our finding that treatment with dopamine agonists was associated with smaller effects of STN DBS on the value of losses chased. However, neuropsychological evidence suggests that the cognitive functions of distinct fronto-striatal-thalamic circuits are optimised or impaired by different levels of dopamine receptor agonists' modulation in PD (Swainson et al., 2000). Dopamine dysregulation syndrome may be mediated by excessive dopamine receptor agonists activity within circuits linking medial prefrontal and ventral striatal sites (Dagher and Robbins, 2009). From this perspective, the use of dopamine receptor agonists enhances the modulation of that circuitry to the point where it is relatively insensitive to STN DBS.

\section{STN DBS and the neuropsychology of loss-chasing behaviour}

Finally, at the current time, it is unclear which other corticolimbic circuits might be influenced by STN DBS to increase the value of losses chased. Previously, we have shown that decisions to quit the chase are associated with neural signals within the anterior cingulate cortex, anterior insula and caudate nucleus, reflecting the impact of cognitive conflict and visceral arousal on neural circuits that interface rewardrelated information and action selection when making a decision to quit during a run of losing gambles (Campbell-Meiklejohn et al., 2008). Anterior cingulate cortex activity also appears to play a significant role in representing some of the cognitive biases that legitimise continued gambling, while signals within the anterior cingulate cortex following bad outcomes of previous decisions to chase are associated with an increased probability of deciding to quit on when the next opportunity to chase losses arises (Campbell-Meiklejohn et al., 2008).

The STN is linked to a distributed circuit, incorporating the inferior frontal cortex and the pre-supplementary area that exerts inhibitory influence over selected action (Aron et al., 2007; Swann et al., 2011). The anterior cingulate cortex also makes an important contribution to the modulation of STN activity (Ballanger et al., 2009; Chudasama et al., 2003; Nakano et al., 2000; Takada et al., 2001). Additionally, increased activity within the anterior cingulate cortex following STN DBS may mediate inhibitory response problems in PD patients (Campbell et al., 2008) while reductions in the activity of the anterior cingulate cortex may drive problems in verbal learning (Kalbe et al., 2009). STN DBS is also associated with impairments in selection tasks that are known to depend upon functions of the anterior cingulate cortex, such as the Stroop test of selective attention (Alegret et al., 2004; Carter et al., 2000; Heo et al., 2008; Klempirova et al., 2007). We propose that STN DBS in PD patients further disrupts the functioning of a circuit incorporating the anterior cingulate cortex and STN (Levy et al., 1997) that tags the reward values of candidate behaviours (Rushworth et al., 2004). In the context of the loss-chasing behaviour exhibited by PD patients, STN DBS increases the incentive value of accumulated losses helping to sustain continued play.

In summary, we have found that STN DBS increased the value of losses chased by PD patients during runs of losing outcomes. Our data also show that these effects are more pronounced in female compared to male patients but reduced in patients taking dopamine receptor agonists or anti-depressant medications. Additionally, the effects of STN DBS on loss-chasing were evident in the second occasion suggesting that either loss chasing increased once patients had become acquainted with the gambling behaviour or, more likely, loss chasing occurs as a predominantly acute effect off STN DBS and slowly vanishes during long-term stimulation. Further studies are needed to clarify this issue.

\section{Acknowledgments}

This research was funded, in part, by independent awards from the Biotechnology and Biological Sciences Research Council and the Medical Research Council (United Kingdom) to Professor Robert D. Rogers and by the German Parkinson's Disease Association (Deutsche Parkinson Vereinigung e.V.) to Dr. Lars Wojtecki and Prof. Alfons Schnitzler. A.S. also acknowledges support from the ERANET-Neuron Grant "PhysioDBS" (Neuron-48-013) and the "Helmholtz Alliance for Mental Health in an Aging Society" (HelMA).

\section{Disclosure statement for authors}

All authors declare no actual or potential conflict of interest including any financial, personal or other relationships with other people or organisations within 3 years of beginning the work submitted. 


\section{Appendix A. Supplementary data}

Supplementary data to this article can be found online at doi:10. 1016/j.expneurol.2011.06.007.

\section{References}

Alegret, M., Valldeoriola, F., Marti, M., Pilleri, M., Junque, C., Rumia, J., et al., 2004 Comparative cognitive effects of bilateral subthalamic stimulation and subcutaneous continuous infusion of apomorphine in Parkinson's disease. Mov. Disord. 19 (12), 1463-1469 (Dec).

American Psychiatric Association, 2000. Diagnostic and Statistical Manual of Mental Disorders DSM-IV-TR Fourth Edition (Text Revision). American Psychiatric Publishing, Inc., Washington, DC.

Ardouin, C., Voon, V., Worbe, Y., Abouazar, N., Czernecki, V., Hosseini, H., et al., 2006. Pathological gambling in Parkinson's disease improves on chronic subthalamic nucleus stimulation. Mov. Disord. 21 (11), 1941-1946 (Nov).

Aron, A.R., Behrens, T.E., Smith, S., Frank, M.J., Poldrack, R.A., 2007. Triangulating a cognitive control network using diffusion-weighted magnetic resonance imaging (MRI) and functional MRI. J. Neurosci. 27, 3743-3752.

Ballanger, B., van Eimeren, T., Moro, E., Lozano, A.M., Hamani, C., Boulinguez, P., et al., 2009. Stimulation of the subthalamic nucleus and impulsivity: release your horses. Ann. Neurol. 66 (6), 817-824 (Dec).

Bandini, F., Primavera, A., Pizzorno, M., Cocito, L., 2007. Using STN DBS and medication reduction as a strategy to treat pathological gambling in Parkinson's disease Parkinsonism Relat. Disord. 13 (6), 369-371 (Aug).

Beck, A.T., Ward, C.H., Medelson, M., Mock, J., Erbaugh, J., 1961. An inventory for measuring depression. Arch. Gen. Psychiatry 4, 561-571

Campbell, M.C., Karimi, M., Weaver, P.M., Wu, J., Perantie, D.C., Golchin, N.A., et al., 2008 Neural correlates of STN DBS-induced cognitive variability in Parkinson disease. Neuropsychologia 46 (13), 3162-3169 (Nov).

Campbell-Meiklejohn, D.K., Woolrich, M.W., Passingham, R.E., Rogers, R.D., 2008 Knowing when to stop: the brain mechanisms of chasing losses. Biol. Psychiatry 63 (3), 293-300 (Feb 1).

Campbell-Meiklejohn, D., Wakeley, J., Herbert, V., Cook, J., Scollo, P., Kar Ray, M., Selvaraj, S., Passingham, R.E., Cowen, P.J., Rogers, R.D., 2010. Serotonin and dopamine play complementary roles in gambling to recover losses: Implications for pathological gambling. Neuropsychopharmacology 36, 402-410.

Carter, C.S., Macdonald, A.M., Botvinick, M., Ross, L.L., Stenger, V.A., Noll, D., et al., 2000. Parsing executive processes: strategic vs. evaluative functions of the anterior cingulate cortex. Proc. Natl. Acad. Sci. U. S. A. 97 (4), 1944-1948 (Feb 15).

Chudasama, Y., Baunez, C., Robbins, T.W., 2003. Functional disconnection of the medial prefrontal cortex and subthalamic nucleus in attentional performance: evidence for corticosubthalamic interaction. J. Neurosci. 23 (13), 5477-5485 (Jul 2).

Corless, T., Dickerson, M., 1989. Gamblers' self-perceptions of the determinants of impaired control. Br. J. Addict. 84 (12), 1527-1537 (Dec).

Creigh-Tyte, S., Lepper, J., 2004. Gender Differences in Participation in, and Attitudes towards, Gambling in the UK: Results from the 2004 NOP Survey.

Cubitt, R., Starmer, C., Sugden, R., 1998. On the validity of the random lottery incentive system. Exp. Econ. 1, 115-131.

Dagher, A., Robbins, T.W., 2009. Personality, addiction, dopamine: insights from Parkinson's disease. Neuron 61 (4), 502-510 (Feb 26).

Dickerson, M., Hinchy, J., Fabre, J., 1987. Chasing, arousal and sensation seeking in offcourse gamblers. Br. J. Addict. 82 (6), 673-680 (Jun)

Driver-Dunckley, E., Samanta, J., Stacy, M., 2003. Pathological gambling associated with dopamine agonist therapy in Parkinson's disease. Neurology 61 (3), 422-423 (Aug 12).

Evans, A.H., Katzenschlager, R., Paviour, D., O'Sullivan, J.D., Appel, S., Lawrence, A.D., et al., 2004. Punding in Parkinson's disease: its relation to the dopamine dysregulation syndrome. Mov. Disord. 19 (4), 397-405 (Apr).

Fahn, S., Elton, R., 1987. UPDRS Development Committee. Unified Parkinson's Disease Rating Scale. In: Fahn, S., Marsden, C., Calne, D., Goldstein, M. (Eds.), Recent Developments in Parkinson's Disease. Macmillan, Florham Park, NJ, pp. 153-163.

Ferrara, J.M., Stacy, M., 2008. Impulse-control disorders in Parkinson's disease. CNS Spectr. 13 (8), 690-698 (August).

First, M.B., Spitzer, R.L., Gibbon, M., Williams, J.B.W., 2002. Structured Clinical Interview for DSM-IV Axis-I Disorders, Research Version, Patient Edition. (SCID-I/P). Biometrics Research, New York.

Frank, M.J., 2006. Hold your horses: a dynamic computational role for the subthalamic nucleus in decision making. Neural Netw. 19 (8), 1120-1136 (Oct)

Frank, M.J., Claus, E.D., 2006. Anatomy of a decision: striato-orbitofrontal interactions in reinforcement learning, decision making, and reversal. Psychol. Rev. 113 (2), 300-326 (Apr).

Frank, M.J., Samanta, J., Moustafa, A.A., Sherman, S.J., 2007a. Hold your horses: impulsivity, deep brain stimulation, and medication in parkinsonism. Science 318 (5854), 1309-1312 (Nov 23).

Frank, M.J., Scheres, A., Sherman, S.J., 2007b. Understanding decision-making deficits in neurological conditions: insights from models of natural action selection. Philos. Trans. R. Soc. Lond. B Biol. Sci. 362 (1485), 1641-1654 (Sep 29).

Hautzinger, M., Bailer, M., Worral, H., Keller, F., 1995. Das Beck Depressions-Inventar (BDI). Huber, Bern.

Heo, J.H., Lee, K.M., Paek, S.H., Kim, M.J., Lee, J.Y., Kim, J.Y., et al., 2008. The effects of bilateral subthalamic nucleus deep brain stimulation (STN DBS) on cognition in Parkinson disease. J. Neurol. Sci. 273 (1-2), 19-24 (Oct 15).
Hoehn, M.M., Yahr, M.D., 1967. Parkinsonism: onset, progression and mortality. Neurology 17 (5), 427-442 (May).

Jahanshahi, M., Ardouin, C.M., Brown, R.G., Rothwell, J.C., Obeso, J., Albanese, A., et al. 2000. The impact of deep brain stimulation on executive function in Parkinson's disease. Brain 123 (Pt 6), 1142-1154 (Jun).

Kahneman, D., Tversky, A., 1979. Prospect theory: an analysis of decisions under risk. Econometrica 47, 263-291.

Kahneman, D., Tversky, A., 2000. Choices Values and Frames. Cambridge University Press, Cambridge, UK.

Kalbe, E., Voges, J., Weber, T., Haarer, M., Baudrexel, S., Klein, J.C., et al., 2009. Fronta FDG-PET activity correlates with cognitive outcome after STN-DBS in Parkinson disease. Neurology 72 (1), 42-49 (Jan 6).

Klempirova, O., Jech, R., Urgosik, D., Klempir, J., Spackova, N., Roth, J., et al., 2007. Deep brain stimulation of the subthalamic nucleus and cognitive functions in Parkinson's disease. Prague Med. Rep. 108 (4), 315-323.

Krack, P., Batir, A., Van Blercom, N., Chabardes, S., Fraix, V., Ardouin, C., et al., 2003. Fiveyear follow-up of bilateral stimulation of the subthalamic nucleus in advanced Parkinson's disease. N. Engl. J. Med. 349 (20), 1925-1934 (Nov 13).

Lawrence, A.J., Blackwell, A.D., Barker, R.A., Spagnolo, F., Clark, L., Aitken, M.R., et al, 2007. Predictors of punding in Parkinson's disease: results from a questionnaire survey. Mov. Disord. 22 (16), 2339-2345 (Dec).

Lesieur, H., 1977. The Chase: Career of the Compulsive Gambler, First ed. Anchor Press/ Doubleday, Garden City, New York.

Lesieur, H.R., 1979. The compulsive gambler's spiral of options and involvement Psychiatry 42 (1), 79-87 (Feb)

Lesieur, H.R., Blume, S.B., 1987. The South Oaks Gambling Screen (SOGS): a new instrument for the identification of pathological gamblers. Am. J. Psychiatry 144 (9), 1184-1188 (Sep).

Levy, R., Hazrati, L.N., Herrero, M.T., Vila, M., Hassani, O.K., Mouroux, M., et al., 1997. Reevaluation of the functional anatomy of the basal ganglia in normal and Parkinsonian states. Neuroscience 76 (2), 335-343 (Jan).

Lim, S.Y., O'Sullivan, S.S., Kotschet, K., Gallagher, D.A., Lacey, C., Lawrence, A.D., et al., 2009. Dopamine dysregulation syndrome, impulse control disorders and punding after deep brain stimulation surgery for Parkinson's disease. J. Clin. Neurosci. 16 (9) 1148-1152 (Sep).

Nakano, K., Kayahara, T., Tsutsumi, T., Ushiro, H., 2000. Neural circuits and functional organization of the striatum. J. Neurol. 247 (Suppl. 5), V1-V15 (Sep).

Okun, M.S., Green, J.R., Saben, R., Gross, R., Foote, K.D., Vitek, J.L., 2003. Mood changes with deep brain stimulation of STN and GPi: results of a pilot study. J. Neurol Neurosurg. Psychiatry 74, 1584-1586.

Pillon, B., Ardouin, C., Damier, P., Krack, P., Houeto, J.L., Klinger, H., et al., 2000 Neuropsychological changes between "off" and "on" STN or GPi stimulation in Parkinson's disease. Neurology 55 (3), 411-418 (Aug 8).

Rosen, A.B., Tsai, J.S., Downs, S.M., 2003. Variations in risk attitude across race, gender and education. Med. Decis. Making 23 (6), 511-517 (Nov-Dec).

Rushworth, M.F., Walton, M.E., Kennerley, S.W., Bannerman, D.M., 2004. Action sets and decisions in the medial frontal cortex. Trends Cogn. Sci. 8 (9), 410-417 (Sep).

Saint-Cyr, J.A., Trepanier, L.L., Kumar, R., Lozano, A.M., Lang, A.E., 2000. Neuropsychological consequences of chronic bilateral stimulation of the subthalamic nucleus in Parkinson's disease. Brain 123 (Pt 10), 2091-2108 (Oct).

Schmidt, R., Freidl, W., Fazekas, F., Reinhart, B., Grieshofer, P., Koch, M., et al., 1994. The Mattis Dementia Rating Scale: normative data from 1,001 healthy volunteers Neurology 44 (5), 964-966 (May).

Schöttle, D., Rode, S., Krüger, S., Bräunig, P., 2006. Selbstbeurteilungsskalen für manische Episoden. Psychiatr. Prax. 33 (Supplementary 1).

Shafir, E., Tversky, A., 1995. Decision making. In: Smith, E.E., Oscherson, D.N. (Eds.) Thinking. MIT Press, Cambridge, MA, pp. 77-100.

Shugar, G., Schertzer, S., Toner, B.B., Di Gasbarro, I., 1992. Development, use, and factor analysis of a self-report inventory for mania. Compr. Psychiatry 33 (5), 325-331.

Smeding, H.M., Goudriaan, A.E., Foncke, E.M., Schuurman, P.R., Speelman, J.D., Schmand B., 2007. Pathological gambling after bilateral subthalamic nucleus stimulation in Parkinson disease. J. Neurol. Neurosurg. Psychiatry 78 (5), 517-519 (May).

Stefurak, T., Mikulis, D., Mayberg, H., Lang, A.E., Hevenor, S., Pahapill, P., et al., 2003. Deep brain stimulation for Parkinson's disease dissociates mood and motor circuits: a functional MRI case study. Mov. Disord. 18 (12), 1508-1516 (Dec).

Swainson, R., Rogers, R.D., Sahakian, B.J., Summers, B.A., Polkey, C.E., Robbins, T.W. 2000. Probabilistic learning and reversal deficits in patients with Parkinson's disease or frontal or temporal lobe lesions: possible adverse effects of dopaminergic medication. Neuropsychologia 38 (5), 596-612.

Swann, N., Poizner, H., Houser, M., Gould, S., Greenhouse, I., Cai, W., Strunk, J., George, J., Aron, A.R., 2011. Deep brain stimulation of the subthalamic nucleus alters the cortical profile of response inhibition in the Beta frequency band: a scalp EEG study in Parkinson's disease. J. Neurosci. 31, 5721-5729.

Takada, M., Tokuno, H., Hamada, I., Inase, M., Ito, Y., Imanishi, M., et al., 2001. Organization of inputs from cingulate motor areas to basal ganglia in macaque monkey. Eur. J. Neurosci. 14 (10), 1633-1650 (Nov)

Tversky, A., Kahneman, D., 1981. The framing of decisions and the psychology of choice. Science 211 (4481), 453-458 (Jan 30).

Tversky, A., Kahneman, D., 1992. Advances in prospect theory: cumulative representation of uncertainty. J. Risk Uncertainty 5 (4), 297-323.

van Eimeren, T., Ballanger, B., Pellecchia, G., Miyasaki, J.M., Lang, A.E., Strafella, A.P. 2009. Dopamine agonists diminish value sensitivity of the orbitofrontal cortex: a trigger for pathological gambling in Parkinson's disease? Neuropsychopharmacology 34 (13), 2758-2766 (Dec). 
Voon, V., Hassan, K., Zurowski, M., Duff-Canning, S., de Souza, M., Fox, S., et al., 2006. Prospective prevalence of pathologic gambling and medication association in Parkinson disease. Neurology 66 (11), 1750-1752 (Jun 13).

Voon, V., Krack, P., Lang, A.E., Lozano, A.M., Dujardin, K., Schüpbach, M., D’Ambrosia, J. et al., 2008. A multicentre study on suicide outcomes following subthalamic stimulation for Parkinson's disease. Brain 131, 2720-2728.

Voon, V., Thomsen, T., Miyasaki, J.M., de Souza, M., Shafro, A., Fox, S.H., et al., 2007. Factors associated with dopaminergic drug-related pathological gambling in Parkinson disease. Arch. Neurol. 64 (2), 212-216 (Feb).

Voon, V., Pessiglione, M., Brezing, C., Gallea, C., Fernandez, H.H., Dolan, R.J., et al., 2010 Mechanisms underlying dopamine-mediated reward bias in compulsive behaviors. Neuron 65, 135-142.

Watson, D., Clark, L.A., Tellegen, A., 1988. Development and validation of brief measure of positive and negative affect: the PANAS scales. J. Pers. Soc. Psychol. 54 (6) 1063-1070 (Jun).
Weintraub, D., Siderowf, A.D., Potenza, M.N., Goveas, J., Morales, K.H., Duda, J.E., et al., 2006. Association of dopamine agonist use with impulse control disorders in Parkinson disease. Arch. Neurol. 63 (7), 969-973 (Jul).

Winstanley, C.A., Baunez, C., Theobald, D.E., Robbins, T.W., 2005. Lesions to the subthalamic nucleus decrease impulsive choice but impair autoshaping in rats: the importance of the basal ganglia in Pavlovian conditioning and impulse control. Eur. J. Neurosci. 21 (11), 3107-3116 (Jun).

Witjas, T., Baunez, C. Henry, J.M. Delfini, M., Regis, J Cherif, A.A et al, 2005. Addiction in Parkinson's disease: impact of subthalamic nucleus deep brain stimulation. Mov. Disord. 20 (8), 1052-1055 (Aug).

Witt, K., Daniels, C., Reiff, J., Krack, P., Volkmann, J., Pinsker, M.O., Krause, M., Tronnier, V., Kloss, M., Schnitzler, A., Wojtecki, L., Bötzel, K., Danek, A., Hilker, R., Sturm, V., Kupsch, A., Karner, E., Deuschl, G., 2008. Neuropsychological and psychiatric changes after deep brain stimulation for Parkinson's disease: a randomised, multicentre study. Lancet Neurol. 7 (7), 605-614. 